\title{
HyperART: non-invasive quantification of leaf traits using hyperspectral absorption-reflectance- transmittance imaging
}

Sergej Bergsträsser ${ }^{1 *}$, Dimitrios Fanourakis ${ }^{1,2,3}$, Simone Schmittgen ${ }^{1}$, Maria Pilar Cendrero-Mateo ${ }^{1}$, Marcus Jansen ${ }^{1,4}$, Hanno Scharr ${ }^{1}$ and Uwe Rascher ${ }^{1}$

\begin{abstract}
Background: Combined assessment of leaf reflectance and transmittance is currently limited to spot (point) measurements. This study introduces a tailor-made hyperspectral absorption-reflectance-transmittance imaging (HyperART) system, yielding a non-invasive determination of both reflectance and transmittance of the whole leaf. We addressed its applicability for analysing plant traits, i.e. assessing Cercospora beticola disease severity or leaf chlorophyll content. To test the accuracy of the obtained data, these were compared with reflectance and transmittance measurements of selected leaves acquired by the point spectroradiometer ASD FieldSpec, equipped with the FluoWat device.

Results: The working principle of the HyperART system relies on the upward redirection of transmitted and reflected light (range of 400 to $2500 \mathrm{~nm}$ ) of a plant sample towards two line scanners. By using both the reflectance and transmittance image, an image of leaf absorption can be calculated. The comparison with the dynamically high-resolution ASD FieldSpec data showed good correlation, underlying the accuracy of the HyperART system. Our experiments showed that variation in both leaf chlorophyll content of four different crop species, due to different fertilization regimes during growth, and fungal symptoms on sugar beet leaves could be accurately estimated and monitored. The use of leaf reflectance and transmittance, as well as their sum (by which the non-absorbed radiation is calculated) obtained by the HyperART system gave considerably improved results in classification of Cercospora leaf spot disease and determination of chlorophyll content.
\end{abstract}

Conclusions: The HyperART system offers the possibility for non-invasive and accurate mapping of leaf transmittance and absorption, significantly expanding the applicability of reflectance, based on mapping spectroscopy, in plant sciences. Therefore, the HyperART system may be readily employed for non-invasive determination of the spatio-temporal dynamics of various plant properties.

Keywords: Absorption, Cercospora beticola, Chlorophyll content, FieldSpec, FluoWat, Hyperspectral imaging, Non-invasive phenotyping, Reflectance, Transmittance, Imaging spectroscopy

\footnotetext{
* Correspondence: sergej.bergstraesser@julumni.fz-juelich.de

${ }^{1}$ Institute for Bio- and Geosciences, IBG-2: Plant Sciences, Forschungszentrum

Jülich $\mathrm{GmbH}, 52425$ Jülich, Germany

Full list of author information is available at the end of the article
} 


\section{Background}

Breeding of stress resistant crops for sustainable agricultural purposes is coupled with the identification of targeted traits and their genetic background [1-4]. In this content, the development and application of non-invasive systems becomes of outmost importance for plant phenotyping applications $[4,5]$. For this purpose, imaging techniques hold a prominent position in estimating various plant characteristics, ranging from pigment content to infection from diseases.

Light arriving on the leaf surface can be reflected, transmitted, or absorbed by the leaf. Quantifying the spectral characteristics of these components, by employing hyperspectral cameras can be very informative for the physiological status of the plants under research. Such spectroscopic retrieval of plant traits is recently gaining attention in the phenotyping community [6,7], not only due to their explicit connection with the leaf function and composition, but also due to the prospect of applying the method as imaging spectroscopy. This methodology offers the acquisition of information for the plants under study, without making physical contact with them. For instance, by using reflectance data, chlorophyll content of Arabidopsis thaliana [8] and disease symptoms' incidence of Beta vulgaris [9-13] were recently monitored. Previous studies, however, are mostly limited to find optimal reflectance wavelengths (combination of those in form of indices) or methods that use reflectance signatures related to leaf characteristics such as chlorophyll content, nutritional status, or infection by diseases. This may be explained by the limited possibilities to measure transmittance in a rapid fashion. Despite diverse attempts in this field the technical possibilities still stay unexhausted. Measurements based only on reflectance or only on transmittance obviously neglect a part of the existing spectral information. Currently there are no known imaging approaches that can measure reflectance and transmittance simultaneously and derive absorption of plant leaves. We hypothesize that plant properties will be better estimated when both reflectance and transmittance are taken into account. Their simultaneous quantification will be highly advantageous for academic and industry-oriented research purposes as demonstrated in our results presented below.

Currently available instruments to quantify hyperspectral plant reflectance and transmittance (e.g., integrating sphere and leaf clip) operate in the spot scale (i.e., a few $\mathrm{cm}^{2}$ ) [14-16]. Therefore, several spots per leaf need to be assessed, in order to have an average value that is representative for the whole leaf. In this way, scaling plant reflectance and transmittance measurements to the entire leaf level will enable a more comprehensive assessment of a larger number of plants. More importantly, whole-leaf measurements offer spatial resolution allowing non-invasive observation of changes in leaf tissue owing to stressors.
Here we present a novel tailor-made instrument, named HyperART (Hyper = hyperspectral and ART Absorption, Reflectance, Transmittance), which quantifies simultaneously reflectance and transmittance at the whole-leaf scale. Firstly, we compare the data, obtained by the newly-introduced equipment, with a wellestablished high-performance spot-scale device (ASD- FieldSpec equipped with FluoWat; $[17,18])$. The FluoWat leafclip provides the possibility to record both reflection and transmission spectra of a single-point (i.e., limited in size) on a leaf. Secondly, we evaluated whether Cercospora beticola disease progression, a wide-spread pathogen, is more accurately derived by using the Cercospora Leaf Spot Index (CLSI) calculated from both reflectance and transmittance, as compared to the estimation that is solely based on reflectance. The CLSI index is computed by using wavelengths that were identified to be sensitive to the $C$. beticola disease symptoms [13]. Further, we tested the additional advantage of the HyperART in the estimation of leaf chlorophyll content of four species (i.e., maize, barley, rapeseed, tomato), based on twenty two commonly-used optical indices.

This paper introduces a novel tailor-made device, called HyperART. It can measure simultaneously spatially- and spectrally- resolved reflection and transmission, allowing the calculation of the spectral absorption image. This additional layer of information is expected to improve the quantification of plant traits. The above-mentioned measurements were performed with a comparable accuracy as with clip-on devices. The HyperART system also provides spatial information that can be potentially used to quantify spatial or temporal dynamics of leaf tissue changes. This is important as almost all processes or changes occurring in a leaf do not take place simultaneously throughout the whole tissue, but have initial points, from where they start spreading. The two employed case studies highlight the possibilities and advantages of the instrument. These are mainly the improved accuracy of results and the upscaling from the spot to the leaf level, encouraging its employment in future phenotyping studies.

\section{HyperART a novel measurement system}

The HyperART system is a novel imaging system developed for non-invasive evaluation of leaf properties, based on recordings of reflectance, transmittance and absorption. In contrast to commercially-available instruments that allow point measurements (i.e., limited to only few $\mathrm{cm}^{2}$ ), this new equipment offers mapping of the whole leaf. For highest precisions, a geometrically precise system and flat samples deserve careful consideration, as described below.

Currently, two prototypes of the HyperART system are available (Figure 1a, b). The first prototype (Figure 1a) was used for the first case study (sugar beet), while the second prototype (Figure 1b) was employed for the measurements of the second case study (rapeseed, tomato, 

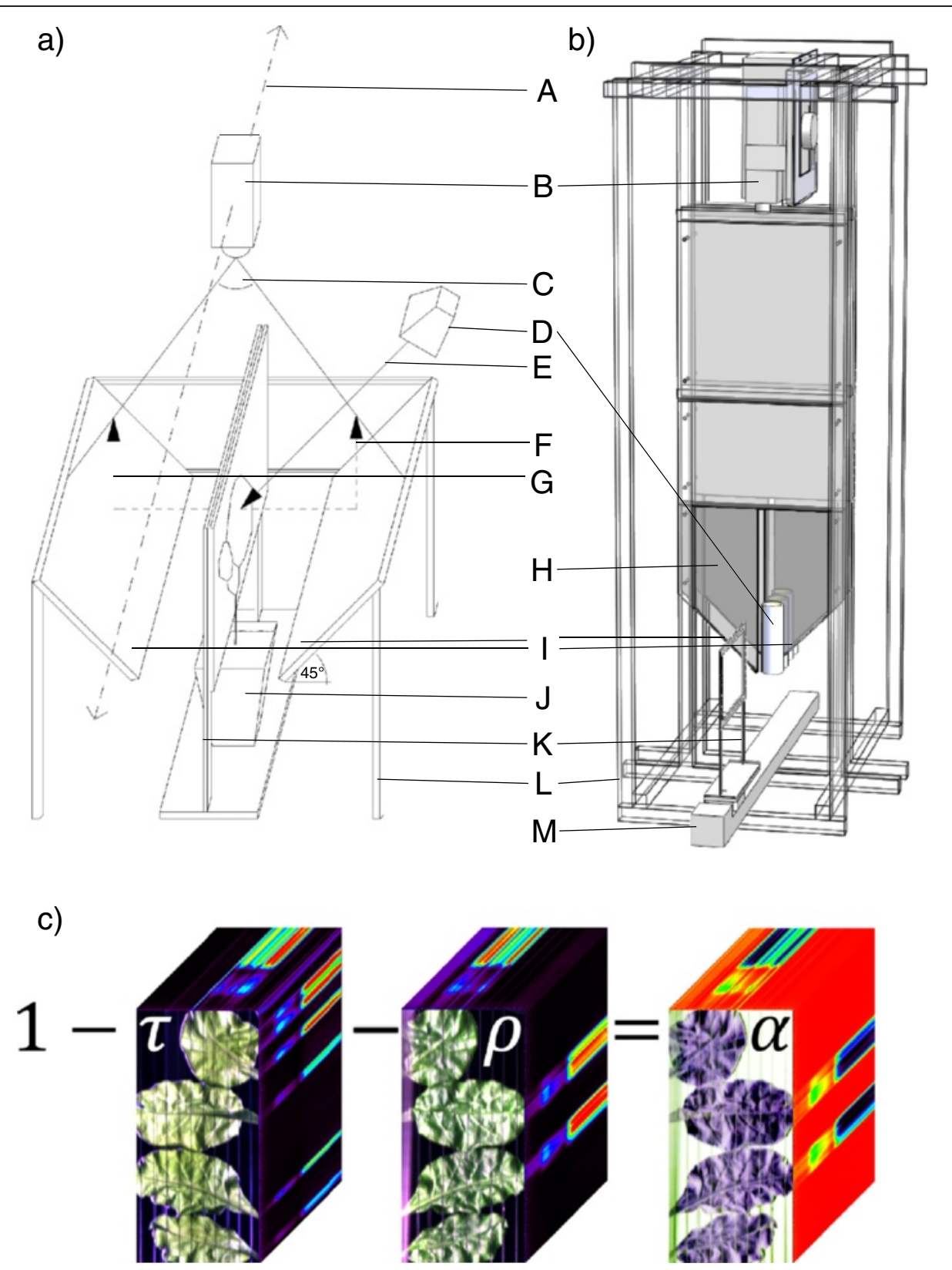

Figure 1 Two prototypes of the HyperART system with an image example. $(\mathbf{a}, \mathbf{b})$ Schematics of the hyperspectral absorption reflectance transmittance imaging (HyperART) system, employed for simultaneous recording of both reflectance $(\rho)$ and transmittance $(\tau)$. In the first prototype (a) the camera is moving, whereas in the second one (b) the samples are moved. A, scan direction; B, line scanner; $C$, field of view; $D$, illumination source; $E$, light beam; $F$, reflected light; $G$, transmitted light; $H$, black painted metal sheets (to avoid direct illumination of the mirror from the light source and to reduce light scattering in the scanning process); I, mirrors; J, plant, of which leaves are fixed in the clip; K, clip, where leaf sample is placed; $L$, framework; M, slide bar. (c) Calculation of absorption (a) in the hyperspectral cube (acquired by the HyperART system), based on $\rho$ and $\tau$.

maize and barley). The main components of both prototypes include: i) a custom-made rectangular clip to hold and flatten the leaves (Figure 1K), ii) two mirrors which redirect both the reflected and transmitted light (Figure 1I), iii) an illumination system (Figure 1D), iv) an imaging system, composed by two hyperspectral cameras (together offering a spectral range of $400-2500 \mathrm{~nm}$; Figure 1B), and v) a slide bar which moves the imaging system (first prototype; Figure 1a A) or the custom-made rectangular clip holding the sample (second prototype; Figure $1 \mathrm{~b}$ $\mathrm{M})$. The main difference between the two prototypes relies on the position of the light source (relative to the mirror) and the scanning procedure (i.e., movement of the imaging system or the clip). The different components of the HyperART system will be described firstly, followed by its working principle. 


\section{Custom-made rectangular clip}

It consisted of two frames that were connected at the top (Figure $1 \mathrm{~K}$ ). The frames had dimensions of length $x$ width $\times$ depth equal to $32 \times 41 \times 0.5 \mathrm{~cm}$ (inner dimensions $28 \times 20 \mathrm{~cm}$ ), and can host samples as large as $560 \mathrm{~cm}^{2}$. The clip keeps the leaf samples as flat as possible. In the first prototype, the frames were made from metal. Moreover, a metal wire, at a distance of $1.5 \mathrm{~cm}$ in the horizontal direction of the frame, was used to position the leaves. The frames and the wire were painted with black mat colour (Dupli Color, MOTIP DUPLI GmbH, Haßmersheim, Germany). In the second prototype, the frames were manufactured from cast polymethylmethacrylat sheet (Plexiglas ${ }^{\circ} \mathrm{GS}$, Evonik Industries AG, Essen, Germany), and were also painted with black mat colour (described above). Leaf positioning was performed by tight black fishing lines at a distance of $2 \mathrm{~cm}$ in both horizontal and vertical (frame) directions.

\section{Mirror}

A flat surface mirror (gertenbach.info e. K., Wölltein, Germany) was employed, having a minimum of $85 \%$ spectral reflectance performance (in the range of 400-700 nm) at $45^{\circ}$ angle relative to illumination. The first prototype contains two (rectangular) mirrors with dimensions of length $\times$ width $\times$ depth equal to $28 \times 20 \times 0.5 \mathrm{~cm}$. The second prototype employs four (rectangular) mirrors with the following dimensions: length $\times$ width $\times$ depth equal to $25.5 \times 0.5 \times 0.3 \mathrm{~cm}$. In both prototypes, the mirrors were placed exactly at $45^{\circ}$ relative to both the leaf clip (thus the leaf sample) and camera planes.

\section{Illumination system}

In the first prototype, sample illumination was offered by two double-ended halogen lamps (OMNILUX ${ }^{\odot}$ 230 V/4000 W R7s 118 mm, OMNILUX, Waldbüttelbrunn, Germany), placed in a halogen spotlight reflector. In the second prototype, similar lamps (OMNILUX 230 V/120 W R7s 117 mm, OMNILUX, Waldbüttelbrunn, Germany) were placed into a custom made tube reflector from a polished-sheet metal. The lamps were covered with frosted quartz glass, which diffuses the light in order to avoid shadows and enhance the quality of the acquired hyperspectral data. A custom-made converter was employed to transform (rectify) alternating current to direct current (residual ripple $\leq 5 \%)$. This was a three-phase power supply unit, manufactured by a company (J.Schneider Elektrotechnik $\mathrm{GmbH}$, Offenburg, Germany). Direct current was preferable for obtaining hyperspectral data by using a line scanner, since it prevents the fluctuation of the halogen lamp emission, which would take place under alternating current. In both prototypes, the lamps were placed in order to illuminate one side of the leaves.

\section{Hyperspectral imaging system}

The HyperART system hosted two hyperspectral cameras employed on sequence, and together offering a spectral range between 400 and $2500 \mathrm{~nm}$. Both devices work as push broom line scan cameras. The first hyperspectral camera (PS V10E, Spectral Imaging Ltd., Oulu, Finland) employs a high speed interlaced CCD detector. It is sensitive in the 400 to $1000 \mathrm{~nm}$ range, and offers a spatio-spectral resolution of $1392 \times 1040$ pixels. The nominal spectral resolution is $2.8 \mathrm{~nm}$ full width half maximum (FWHM). The spectral sampling interval depends on the spectral binning and varies between 0.63 and $5.06 \mathrm{~nm}$. In our experiments with the first prototype, a binning of 4 (spectral resolution) and 1 (spatial resolution) was employed. In the experiments with the second prototype, a binning of two (spectral resolution) by two (spatial resolution) was employed. The second hyperspectral camera (SWIR, Spectral Imaging Ltd., Oulu, Finland) uses a cooled, temperature-stabilized MCT detector, which is sensitive to the spectral range between 1000 and $2500 \mathrm{~nm}$. The sensor can acquire 320 spatial pixels' and 256 spectral bands' images. The spectral sampling rate is $6.3 \mathrm{~nm}$, combined with a nominal spectral resolution of $10 \mathrm{~nm}$ (FWHM).

\section{Scanning procedure}

At the first prototype, the two hyperspectral cameras were fixed on a slide bar (BiSlide, Velmex Inc., Bloomfield, NY, USA) that moves along an $1.5 \mathrm{~m}$ path at a constant speed $\left(\approx 15 \mathrm{~mm} \mathrm{sec}^{-1}\right)$. The cameras were moved in parallel to the middle line and between the opposite standing mirrors. As the hyperspectral cameras were moved along the slide bar, they recorded the reflected and transmitted light of the whole leaf sample, which was placed in the clip, as explained above. In the second prototype, the hyperspectral cameras were fixed on a framework over the middle point and between the opposite standing mirrors (Figure 1b L). In this case, the clip (holding the leaf sample) was moved along the middle line, between the mirrors that were fixed on the slide bar (Figure $1 \mathrm{~b} M$ ).

The working principle of the HyperART system was identical for both prototypes, and enabled the upward redirection of transmitted and reflected light from the leaf sample towards the hyperspectral camera system through the mirrors. Thus, the unique advantage of the HyperART system is that a reflectance and transmittance image of a leaf can be recorded simultaneously by a single hyperspectral camera. For measurements, leaves must be positioned into the clip (Figure $1 \mathrm{~K}$ ), which can be done without excising them from the plant, since the pot $(\leq 17 \mathrm{~cm}$ height) can be placed below the clip frame (Figure 1a J). Placing the leaves between the frames directs the leaf surface into a flat plane. Inside the clip, the leaves were illuminated from one side, while the leaf was positioned between the 
two mirrors (Figure 1I). Each mirror faced a different side of the clip (thus the sample). In the first prototype, the light source was placed behind one mirror, and opposite to the sample at an angle of $45^{\circ}$ (Figure 1a D). In the second prototype, the light source was placed from the sides of mirrors (Figure $1 \mathrm{~b} \mathrm{D}$ ) that allowed nearly direct illumination of the sample. The mirrors posed both the clip window with the sample, and the camera, at a $45^{\circ}$ angle. One mirror redirected the reflected light (Figure 1a F) to the two line scanners (i.e., upwards; Figure 1B), while the other mirror did the same for the transmitted light (Figure 1a G). The distance of the upper mirror edge to the two line scanners was set to $0.85 \mathrm{~m}$ for both prototypes.

To determine the incoming electromagnetic radiation by the first prototype, a 99\% reflective white standard (Spectralon, Labsphere Inc., North Dutton, NH, USA) was placed instead of the clip. This was done before and following the measurements. In the second prototype, the transmitted light was determined by placing a white diffuser lambertian transmission sheet (Zenith Polymer $^{\circ} \approx 50 \%$ transmission, SphereOptics $\mathrm{GmbH}$, Uhldingen, Germany) between the mirrors.

\section{Image processing}

Hyperspectral images were processed by using ENVI 4.8 (Exelis Visual Information Solutions, Inc., Boulder, CO, USA) and MatLab R2012b (MathWorks ${ }^{\circ}$, Natick, Massachusetts, USA) Software. Different protocols were used for image pre-processing obtained from the first and second prototype.

\section{First prototype}

Firstly, a dark frame image was subtracted from both the leaf and white reference images (Fastspec extension of ENVI). Secondly, images were cut to separate the reflection and transmission images. The transmission image was flipped to overlay the reflection image, and co-registered by the corresponding points in order to correct slight distortions, produced by image acquisition (VLFeat toolbox in MatLab; [19]). In the next step, each image pixel was divided by the corresponding pixel within the white reference image, and multiplied by a correction factor for white reference material (provided by Labsphere Inc.), aiming at both correcting for illumination differences and achieving relative values (Spectral Math tool of ENVI).

\section{Second prototype}

In this prototype images needed modified processing steps since the scan procedure was different as compared to the first prototype. Additionally to the white standard, we here measured a white diffuser lambertian transmission sheet, before scanning the plant samples. By mosaicking both white references we got a white reference image with the same numbers of lines as the sample image. The sample image was normalized by this white reference image for reflection and transmission sides line by line using a normalisation tool from SPECIM (Spectral Imaging Ltd., Oulu, Finland) implemented in ENVI. The transmission image was then flipped and coregistered. With the objective to obtain correct relative values, a correction factor was applied to the reflection and transmission images (provided by Labsphere Inc. and SphereOptics GmbH, respectively).

\section{Absorption mapping}

Based on the assumption that the sum of absorption $(\alpha)$, reflectance $(\rho)$ and (flipped) transmittance $(\tau)$ equals to 1 (Figure 2), $\alpha$ was calculated by subtracting $\rho$ and $\tau$ from the unit (i.e., $\alpha=1-\rho+\tau$ ) for images acquired by using both prototypes. The $\alpha$ contains the information about leaf components and is not sensitive to light scattering effects, caused by leaf surface properties [20]. However, it is not practical to apply different vegetation indices on $\alpha$, because these indices were developed for approaches based on $\rho$ measurements. To highlight the advantage of the newly-introduced HyperART system and make the data comparison clear, a $\rho$ comparable spectral signature ought to be employed. Therefore, the non-absorbed radiation $\rho+\tau$ images (that are equal to inverted $\alpha$ because $\rho+\tau=1-\alpha)$ were used in analysis.

\section{Results and discussion}

\section{Validation of HyperART spectral data}

Reflectance and transmittance spectral signatures of sugar beet leaves were recorded by using either the HyperART system or the FieldSpec spectoradiometer, equipped with a FluoWat device (Figure 2a). In order to validate the HyperART-obtained measurements, reflectance ( $\rho$ ), transmittance $(\tau)$, and the sum of reflectance and transmittance spectra (non-absorbed radiation, $\rho+\tau$ ) were compared to the FluoWat spectra (Figure 2).

A similar $\rho$ and $\tau$ spectrum acquired by either device was found (Figure 2a). Over the full $\rho$ spectral range (except 1400-1500 nm), relative values acquired by the HyperART system were 1-5\% lower than those of FluoWat. For the $\tau$ spectra, the HyperART system data yielded either higher $(>800 \mathrm{~nm})$ or lower $(550 \mathrm{~nm})$ values than those of the FluoWat data. However, the difference in the T spectra was minor $(\approx 1 \%)$, as compared to the difference in the $\rho$ spectrum. In the FluoWat device, there was an overlap between the $\rho$ and $\tau$ spectra at the near infrared region (NIR, 736-1100 nm) (Figure 2a). HyperART $\rho$ and $\tau$ spectra were overlapping in the 1012-1031 nm region. Because of this overlap, the normalized $\alpha$ spectrum is not presented. The noted differences between the values, obtained by the two devices, might be due to the fact that the leaf regions measured by the FluoWat device, are not exactly identical as the selected (for comparison) areas in 
a)

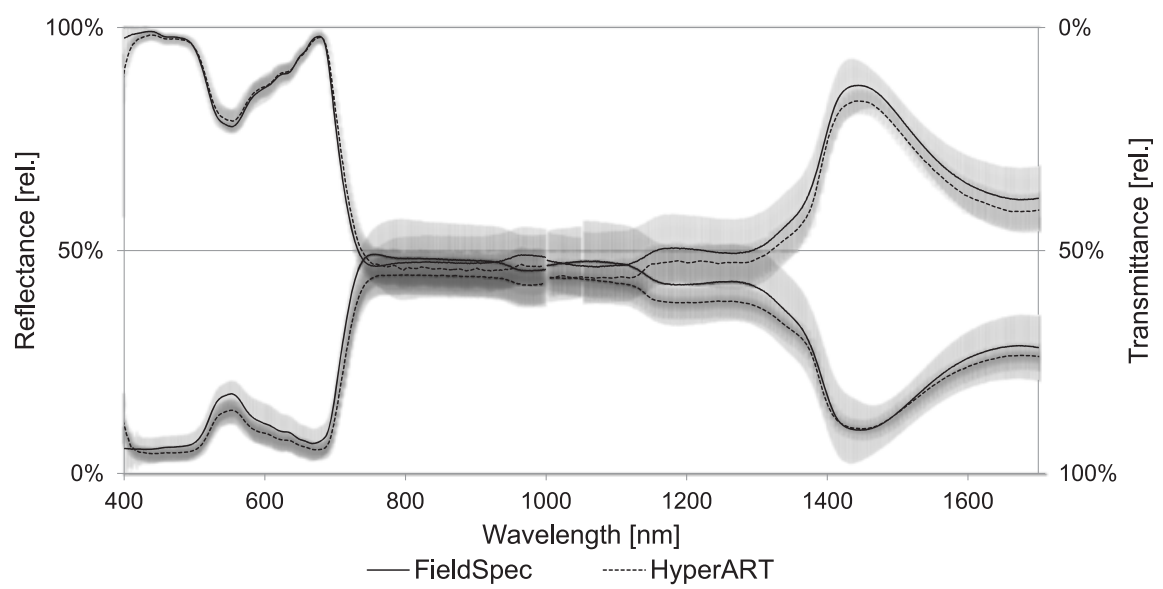

b)

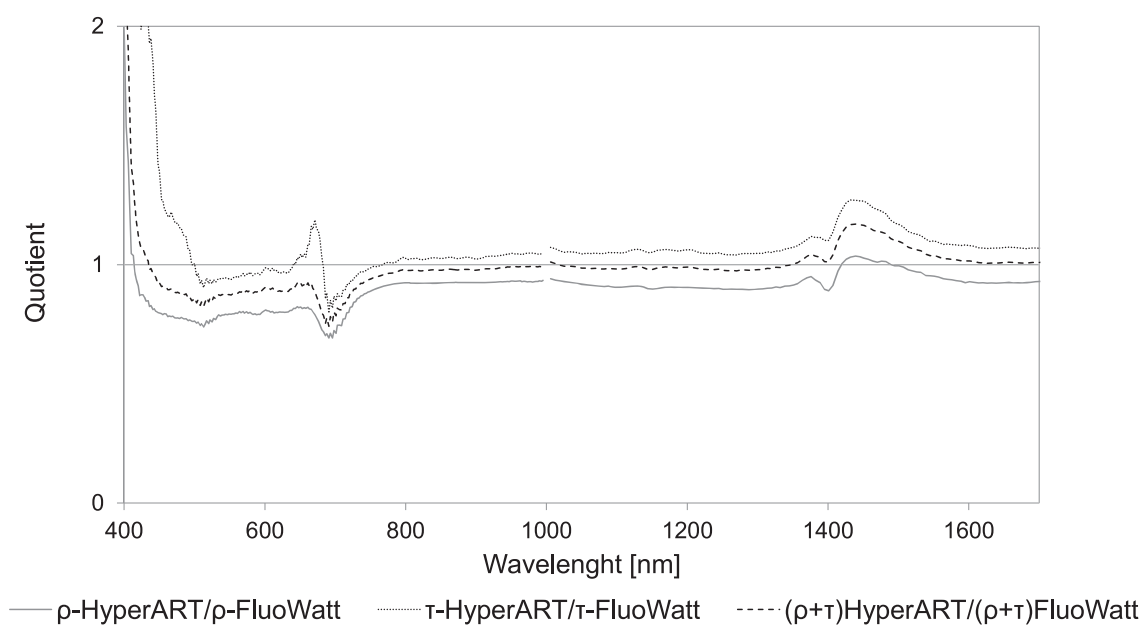

Figure 2 Comparison of spectral signatures obtained by FluoWat and HyperART devices. (a) Reflectance $(\rho)$ and transmittance $(\tau)$ spectra of sugar beet leaves acquired by using FluoWat or the hyperspectral absorption reflectance transmittance imaging (HyperART) system. Data are expressed as relative values. Dashed areas indicate SEM $(n=5)$. (b) Quotients of $\rho, \tau$ and sum of $\rho$ with $\tau$ (i.e., $\rho+\tau)$ of spectral data acquired by using FluoWat and the HyperART system.

the images obtained by using the HyperART system. The gap of the values observed at $1000 \mathrm{~nm}$ at both $\rho$ and $\tau$ spectra, is due to the configuration of the FieldSpec and the hyperspectral imaging system. Both devices combine two different sensors, where the first one covers the region up to $1000 \mathrm{~nm}$, while the second one includes longer wavelengths (i.e., $\geq 1000 \mathrm{~nm}$ ).

For the $\rho, \tau$, and $\rho+\tau$ HyperArt normalized spectra (Figure $2 \mathrm{~b}$ ) the highest deviations were observed in the photosynthetic active radiation region (PAR, 420$730 \mathrm{~nm})$ and around the water absorption band at $1450 \mathrm{~nm}$. For the whole spectrum $(420-1700 \mathrm{~nm})$, standard deviations of $0.07(\rho), 0.19(\tau)$ and $0.07(\rho+\tau)$ were found. These low standard deviation values indicate that the data obtained with the HyperART system are comparable to those taken by using FluoWat.

The coupling of two hyperspectral cameras (i.e., VNIR and SWIR) might be important for future work, where additional traits, from those studied here, are of interest. For instance, the spectral region, offered by the SWIR camera (i.e., 1000-2500 nm), is often employed to detect senescence and water stress symptoms [21,22]. We were able to show that the HyperART system performs well with the SWIR camera, though its potential in detecting plants characteristics is not dealt in the current study. The SWIR images and the VNIR images can be matched together using corresponding points. This matching of VNIR and SWIR images may assist extended spectral signature for every pixel in only one image. However the spatial resolution in the VNIR image needs to be adjusted to the SWIR image or vice versa.

\section{Cercospora beticola symptoms}

Following the data comparison between the HyperART system and FluoWat device we tested if the HyperART is suitable for the detection of Cercospora leaf spot 
disease. Therefore normalized histograms (Figure 3) of the CLSI images with the leaves of one infected and one non-infected sugar beet plant were analysed and compared among each other. Additionally the precision of the unsupervised classification on the $\rho, \tau$ and $\rho+\tau$
CLSI images of the infected leaves were verified (Figure 4).

Three leaves of the infected sugar beet plant (shown in Figure 4) can be described by visual inspection as: i) symptom-free leaf (lower leaf in Figure 4), ii) leaf

a)

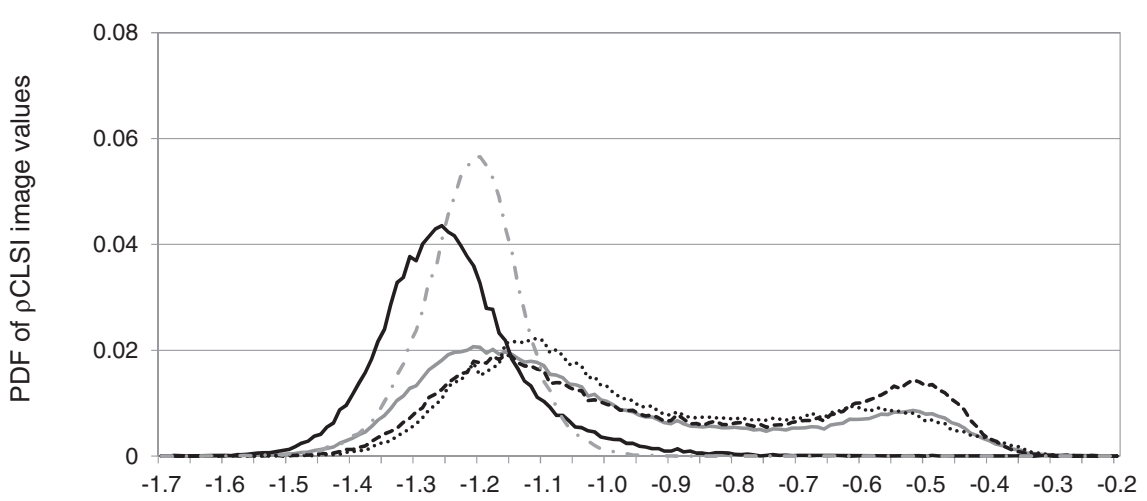

b)

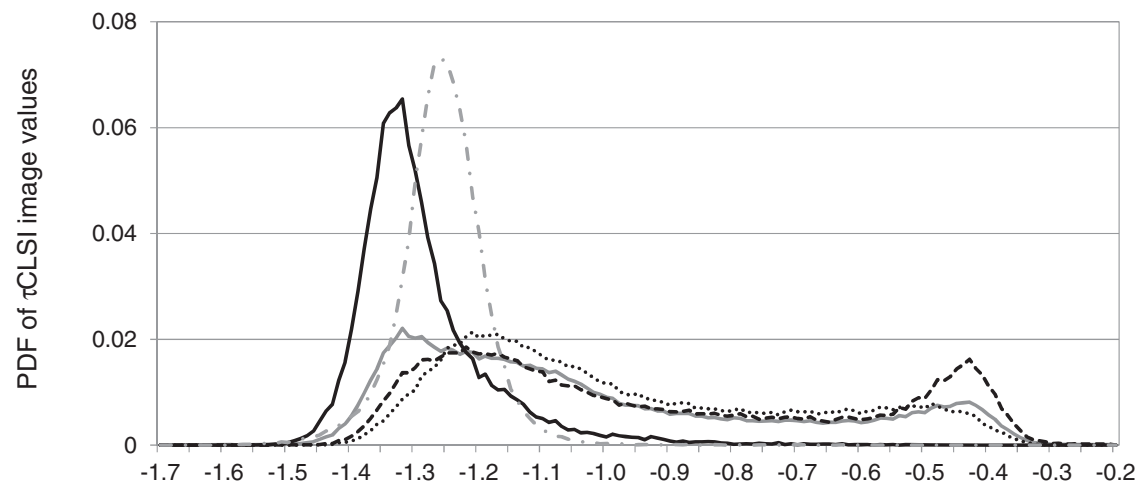

c)

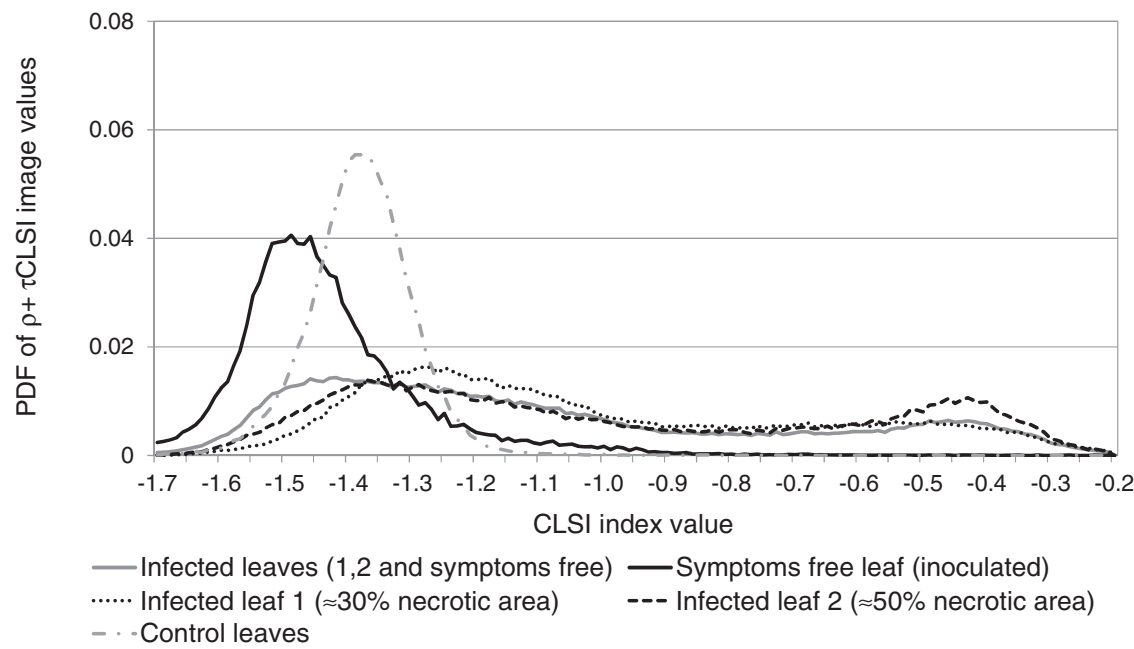

Figure 3 Values difference in normalized histograms of Cercospora Leaf Spot Index (CLSI) images. Normalized histograms (estimated probability functions PDF) of the calculated CLSI values based on (a) reflectance $(\rho)$, (b) transmittance $(\tau)$ or (c) combination of $\rho$ with $\tau$ (i.e., $\rho+\tau)$. Spectra were acquired by using the hyperspectral absorption, reflectance, transmittance (HyperART) imaging system. Measurements were conducted on three leaves of an infected sugar beet plant, and on three leaves of another non-infected (control) sugar beet plant. The two leaves of the former plant showed visual symptoms of infection, whereas the third one was symptom-free. 

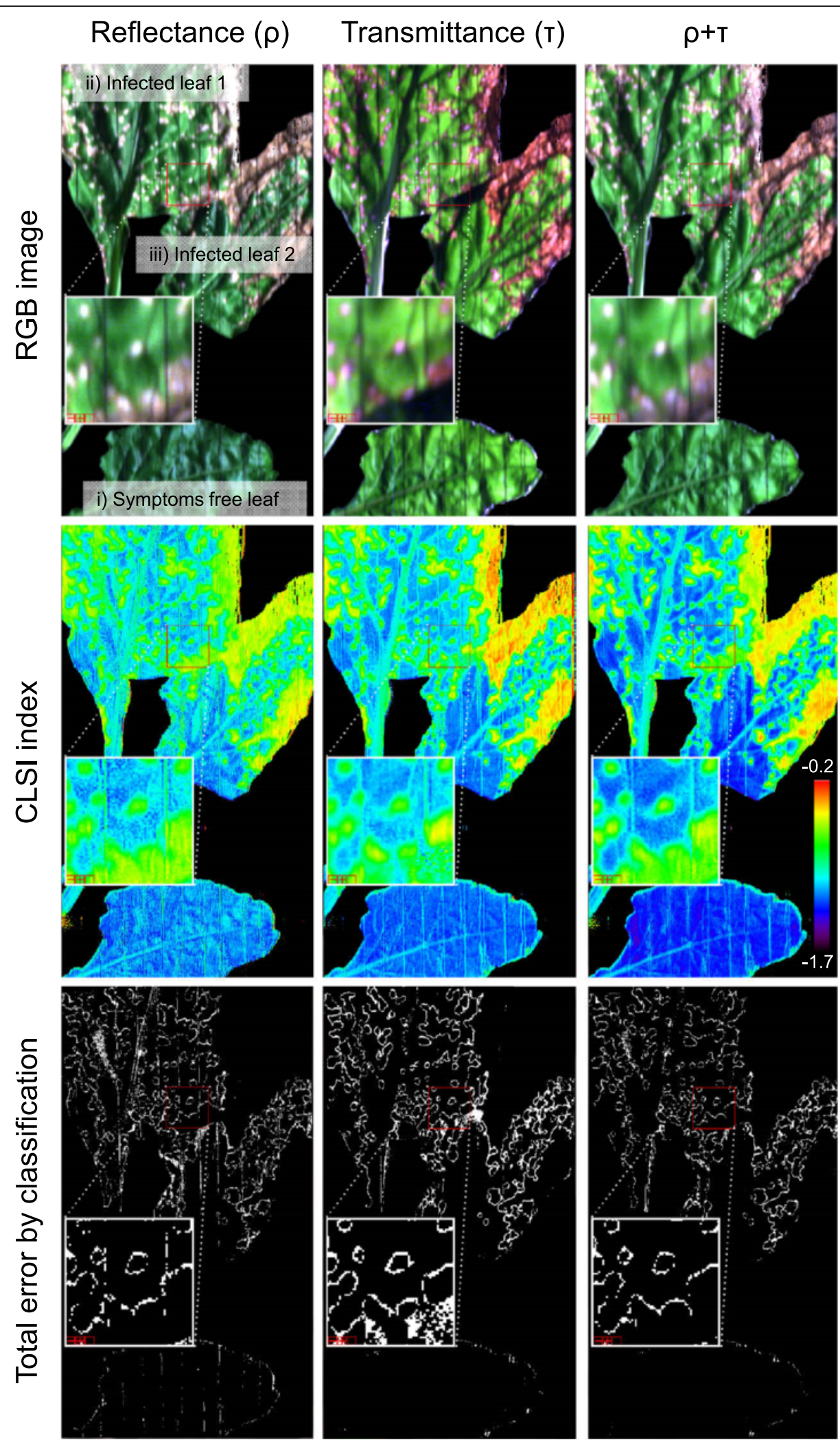

Figure 4 (See legend on next page.) 
(See figure on previous page.)

Figure 4 RGB, colour coded Cercospora Leaf Spot Index (CLSI) and total classification error images. Images were calculated from reflectance $(\rho)$, transmittance $(\tau)$ and combination of $\rho$ with $\tau$ (i.e., $\rho+\tau$ ) of sugar beet leaves infected by Cercospora beticola. The classification was performed by supervised SVM (support vector machine) classification on hyperspectral images, and unsupervised K-Mean (on CLSI images) using two classes (visible symptoms and plat tissue without visible symptoms). Images were acquired by using the hyperspectral absorption reflectance transmittance imaging (HyperART) system. The rectangular panels in the images show the enlargement of red bordered infected leave surface.

with $\approx 30 \%$ infected area (upper leaf in Figure 4), and iii) leaf with $\approx 50 \%$ necrotic area (middle leaf in Figure 4 ). In order to assess the CLSI value changes induced by pathogen infection, the CLSI histogram from three leaves of the non-infected control plant was also computed.

The CLSI shows similar bimodal distribution when calculated from $\rho$ ( $\rho$ CLSI), $\tau$ ( $\tau$ CLSI), or $\rho+\tau(\rho+\tau C L S I)$ images of leaves with infection symptoms. The normalized histogram of the leaves attached on the control plant, or the symptom-free leaf, attached on the infected plant, were clearly different (Figure 3 ). Furthermore there is a difference between the normalized histogram of inoculated but still symptom-free leaf and the normalized histogram of control leaves. They both show a single maximum only, however, the distribution of the symptom-free leaves show a heavy right tail, whereas the control leaves are normally distributed. As a right tail is an index shift towards infection symptoms, this indicates that pre-symptomatic fungal development changed leaf spectral properties. Therefore the CLSI offers a perspective for using the method in early detection of fungal infection stages.

The normalized histograms of the symptomatic leaves showed a shift from the right peak that is related to the degree of leaf infestation. While the normalized $\rho$ and $\rho+\tau$ histograms are Gaussian-like, the normalized $\tau$ histograms were sharper, indicating a lower noise influence. Peak locations varied for the different measurement modalities. The distance between the peak locations were larger in TCLSI and largest in $\rho+$ TCLSI compared to $\rho C L S I$. This might enable a better assignment of the pixels into the categories infected or non-infected. The bimodal amplitude $\left(\mathrm{A}_{\mathrm{b}}\right)$ was equal to $0.45,0.48$ and 0.43 for $\rho$ CLSI, $\tau$ CLSI and $\rho+\tau C L S I$, respectively, being in accordance with our observation of sharper peaks in the normalized tCLSI-histogram. Considering all CLSI histograms, it can be stated that over $90 \%$ of the pixels in the index images were distributed between -1.7 and -0.2 . Higher values indicate increasing disease severity and vice-versa.

The computed CLSI value range in this study differs due to variation in plant properties and measurement method from the range $(-0.45$ to 0.3$)$ reported in the literature $[13,23]$. For instance, leaf pigment content, as well as leaf internal and external structure have been shown to affect plant reflectance properties [24]. In addition, illumination conditions (e.g., shadow or illumination angle) during measurement or measurement methodology (e.g., point measurements, leaf scale or canopy scale) can also raise differences in the CLSI values' range. The differences in CLSI value may be explained by the use of $698 \mathrm{~nm}$ and $734 \mathrm{~nm}$ wavelengths in CLSI equation. These wavelengths are located in the red edge spectral region, and are sensitive to chlorophyll content [25-28]. The sensitivity at $698 \mathrm{~nm}$ is translated to decreased reflectivity (thus lower CLSI values in the normalized part of equation), as chlorophyll content increases. When we subtract lower reflectance value (due to chlorophyll absorption at $734 \mathrm{~nm}$ ) from the normalised part of equation then this might produce slightly higher CLSI values of the leaves attached on the control plant, as compared to the symptom-free leaf attached to the infected plant.

To investigate the classification performance, the tissue having CLSI values lower than the lower peak center was categorized as 'non-infected' $(\approx 27 \%, 14 \%$, and $21 \%$ of the pixels for $\rho$ CLSI, $\tau$ CLSI, and $\rho+\tau$ CLSI, respectively), while tissue giving higher values than the higher peak center is termed as 'infected' $(\approx 14 \%, 19 \%$, and $36 \%$ of the pixels for $\rho C L S I, \tau C L S I$, and $\rho+\tau C L S I$, respectively). The remaining pixels $(\approx 59 \%, 67 \%$, and $43 \%$ for $\rho C L S I, T C L S I$, and $\rho+\tau C L S I$, respectively) between the peaks allow the presence of classification errors. This observation leads to the conclusion that the separation of infected from noninfected leaf tissue is better done with the $\rho+$ TCLSI image, since most of the tissue area is unambiguously classified.

In the RGB $\tau$ image, the reddish colour may be an indication of anthocyanin production in the (sugar beet) leaves, due to infection (Figure 4). The CLSI images have a rainbow colour code, ranging from -1.7 (blue) to -0.2 (red) (Figure 4), which is similar to the histograms in Figure 3. The $\rho$ CLSI image seems to have a slightly higher noise level, as compared to the other images. The TCLSI image shows a better contrast between extremes, while the $\rho+\tau C L S I$ image differentiates the initial symptom and its growing borders. The unsupervised classification yields $93.4 \%, 92.3 \%$ and $94.7 \%$ overall accuracy (automatically calculated in ENVI using contingency matrix which did compare supervised classification with unsupervised classification) for the whole $\rho$ CLSI, $T$ CLSI and $\rho+\mathrm{TCLSI}$ images respectively, being well in accordance with our previous observation. In total, the $\rho$ CLSI index classification quantifies $33.9 \%$ of the tissue as infected (i.e., $66.1 \%$ non-infected), while the TCLSI index 
classification led to an estimation of $29.3 \%$ infected tissue area (i.e., $70.7 \%$ non-infected). The $\rho+\tau$ CLSI image classification results in $31.8 \%$ infected leaf area, and gave the best overall accuracy, as explained below.

The total classification error images (Figure 4) suggest that the noise in the $\rho$ CLSI image leads to an overestimation of infected area. This overestimation may be due to the leaf veins or shadow parts, as a result of leaf waviness. Another error source is an overlay of leaves by the black wire taut in the leaf clip. The opposite is noted for the TCLSI image classification, where the non-infected area seems to be overestimated. This overestimation of the non-infected area might be due to both the overlapping region between the two leaves, which were together placed in the clip, and an underestimation of the symptom border. The TCLSI was found to feature comparably low noise, being also in accordance with the sharper peaks in the respective histogram (Figure 3). The $\rho+\tau$ TLSI image gave the best class separation, supressed noise and counted overlapping regions that led to the most precise estimation of disease severity. In the $\rho+\tau C L S I$ image, the unsupervised classification gave a $99.4 \%$ of non-infected tissue area in the symptom-free leaf. The infected leaves were classified to have $38.7 \%$ and $44.6 \%$ infected areas, respectively.

In general, our visual estimation had an error between $5.4 \%$ and $8.7 \%$, as compared to the image classification results. Therefore, the machine-based classification method (in our case the HyperART system) was found to be more precise and reliable in quantifying disease severity, as compared to assessments based on visual inspection [29].

\section{Leaf chlorophyll content estimation}

Leaves of four species (maize, rapeseed, barley and tomato), grown under two different nitrogen regimes, were analysed for chlorophyll content. The relationships between 22 optical indices (summarized in Table 1), calculated from 3 source HyperART images $(\rho, \tau$, and $\rho+\tau)$ and destructively-assessed chlorophyll content were analysed (i.e., 66 indices $=22$ optical indices $\times 3$ HyperART source images). Both logarithmic (Table 2) and linear (Table 3) regression models were employed for the analysis.

Out of 66 indices, a significant and positive correlation $\left(P<0.001 ; R^{2} \geq 0.5\right)$ between calculated chlorophyll content and destructively measured content was found in 25 and 18 indices for logarithmic and linear regression, respectively (bold highlighted in Tables 2 and 3 ). Importantly, 23 (out of the 25) and 17 (out of the 18) indices for logarithmic and linear regressions, respectively, were computed from $\tau$ and $\rho+\tau$ source images (bold italic highlighted in Tables 2 and 3). These results indicate that the additional $\mathrm{\tau}$ measurements per se or in combination with $\rho$ (i.e., $\rho+\tau$ ) result in higher correlation coefficients between measured and estimated chlorophyll content.
Logarithmic regressions always gave higher correlation coefficients, as compared to linear regression models (Tables 2 and 3). Previous work also reports that indices are non-linearly related to chlorophyll content [31]. The highest correlation coefficients (i.e., $0.63 \leq \mathrm{R}^{2} \leq 0.72$ ) were noted in the logarithmic regression models of the following five indices: $\rho+\tau E V I$, TmND, $\rho+\tau m N D$, TVOG, and $\rho$ BGI2 (Table 2, and Additional file 1: Figure S1). Importantly, the good correlation of these five indices with the destructively-measured values was driven by differences in chlorophyll concentration, owing to both the growth nitrogen regime and species.

Indices developed for carotenoids' pigment changes (i.e., SIPI, PSRI) showed a poor relationship with chlorophyll content (i.e., $\mathrm{R}^{2} \leq 0.52$; Tables 2 and 3). Indices, traditionally used for vegetation monitoring (i.e., NDVI, $\mathrm{SR}, \mathrm{mSR}$ ), did not obtain as good results as red edge and combined indices (Tables 2 and 3). For NDVI specifically, its low performance might be explained by the fact that it changes only when chlorophyll concentration do not exceeds the value of $10 \mu \mathrm{g} \mathrm{cm}^{-2}$ [31,46]. In this study, chlorophyll concentration was eight- to nine-fold higher than this value. Indices developed for maximal sensitivity to leaf area index (i.e., MACRI, TCARI, TCARI/ OSAVI) presented low correlation coefficients (i.e., $\mathrm{R}^{2} \leq$ 0.44; Tables 2 and 3). This is because the aforementioned indices are not sensitive to changes in chlorophyll content at low leaf area index values [43], as those of this study where a set of a few leaves were assessed.

Interestingly, indices based on the off-chlorophyll absorption centre wavebands (690-730 nm; e.g., mND, VOG) seemed to perform better than indices based on the main chlorophyll and carotenoids' absorption regions (450-550 and 670-680 nm, respectively; e.g., NDVI, MCARI) [47,48].

Besides the optical indices, employed here, there are also other approaches to estimate chlorophyll content by using spectral signature [49-51]. In this paper, we look into the first methodology, due to the simplicity of computation and implementation in studying plant properties. However, the performance of the HyperART, when using the aforementioned more elaborate approach, stays unexhausted and necessitates further work.

\section{Conclusion}

The HyperART system was designed to provide data with high spectral and spatial resolution, which can be readily employed for phenotypic measurements, such as disease detection and quantification of chlorophyll content. The newly-introduced HyperART system scans entire leaves, attached to the plants, whereas currently-used instruments (e.g., FluoWat) are limited to spot (point) measurements. The system measures both reflectance $(\rho)$ and transmittance $(\tau)$ data, by which the absorption $(\alpha)$ spectrum can be calculated, with one sensor. In this study we 
Table 1 Formulas and abbreviations of the employed vegetation indices

\begin{tabular}{|c|c|c|c|}
\hline Index & Equation & Indicator (Scale) & Reference \\
\hline $\begin{array}{l}\text { Area under continuum- } \\
\text { removed curve }\end{array}$ & ANCB $_{650-720}:$ Continuum removal based method & Chlorophyll a \& b content (Canopy) & {$[30]$} \\
\hline Blue/Green index & $\mathrm{BGI}_{2}=\frac{\mathrm{R} 450}{\mathrm{R} 550}$ & Chlorophyll content (Canopy) & [31] \\
\hline Chlorophyll green index & Chlgreen $=\frac{R 790}{R 550}-1$ & Chlorophyll (Leaf) & {$[32]$} \\
\hline Chlorophyll red edge index & Chlred edge $=\frac{R 790}{R 705}-1$ & Chlorophyll (Leaf) & {$[32]$} \\
\hline Enhanced vegetation index & $\mathrm{EVI}=2.5 * \frac{\mathrm{R} 800-\mathrm{R} 670}{\mathrm{R} 800+6 * \mathrm{R} 670-7.5 * \mathrm{R} 400+1}$ & Chlorophyll (Canopy) & {$[33]$} \\
\hline $\begin{array}{l}\text { Modified chlorophyll } \\
\text { absorption reflectance index }\end{array}$ & MCARI $=((\mathrm{R} 701-\mathrm{R} 670)-0.2 *(\mathrm{R} 701-\mathrm{R} 550)) * \frac{\mathrm{R} 701}{\mathrm{R} 670}$ & Chlorophyll (Leaf, Canopy) & [34] \\
\hline $\begin{array}{l}\text { Modified normalized } \\
\text { difference index }\end{array}$ & $\mathrm{mND}=\frac{\mathrm{R} 750-\mathrm{R} 705}{\mathrm{R} 750+\mathrm{R} 705-2 * \mathrm{R} 445}$ & Chlorophyll (Leaf) & {$[35]$} \\
\hline Modified simple ratio & $m S R=\frac{R 750-R 445}{R 705-R 445}$ & Green biomass Chlorophyll (Leaf) & {$[35]$} \\
\hline Normalized difference index & $N D=\frac{R 750-R 705}{R 750+R 705}$ & Chlorophyll (Leaf) & {$[36]$} \\
\hline $\begin{array}{l}\text { Normalized difference } \\
\text { vegetation index }\end{array}$ & $\mathrm{NDVI}=\frac{\mathrm{R} 800-\mathrm{R} 670}{\mathrm{R} 800+\mathrm{R} 670}$ & Biomass, leaf area (Canopy) & {$[37]$} \\
\hline $\begin{array}{l}\text { Structure insensitive pigment } \\
\text { index }\end{array}$ & $\mathrm{SIPI}=\frac{R 800-R 455}{\mathrm{R} 800+\mathrm{R} 680}$ & Carotinoid/chlorophyll a ratio (Leaf) & {$[38]$} \\
\hline Simple ratio 1 & $\mathrm{SR}_{750 / 710}=\frac{\mathrm{R} 750}{\mathrm{R} 710}$ & Chlorophyll (Canopy) & {$[39]$} \\
\hline Simple ratio 2 & $S R=\frac{R 800}{R 670}$ & Green biomass (Canopy) & [40] \\
\hline $\begin{array}{l}\text { Pigment specific normalized } \\
\text { difference a }\end{array}$ & PSNDa $=\frac{R 800-R 680}{R 800+R 680}$ & Chlorophyll a (Leaf) & [41] \\
\hline $\begin{array}{l}\text { Pigment specific normalized } \\
\text { difference } b\end{array}$ & $P S N D b=\frac{R 800-R 635}{R 800+R 635}$ & Chlorophyll b (Leaf) & [41] \\
\hline Plant senescence index & $P S R I=\frac{R 680-R 500}{R 750}$ & Plant senescence (Leaf) & [42] \\
\hline Pigment specific simple ratio a & PSSRa $=\frac{R 800}{R 680}$ & Chlorophyll a (Leaf) & [41] \\
\hline Pigment specific simple ratio b & $\mathrm{PSSRb}=\frac{\mathrm{R} 800}{\mathrm{R} 635}$ & Chlorophyll b (Leaf) & [41] \\
\hline $\begin{array}{l}\text { Transformed chlorophyll } \\
\text { absorption in reflectance } \\
\text { Index }\end{array}$ & $\mathrm{TCARI}=3 *\left((\mathrm{R} 700-\mathrm{R} 670)-0.2 *(\mathrm{R} 700-\mathrm{R} 550) *\left(\frac{\mathrm{R} 700}{\mathrm{R} 670}\right)\right)$ & Chlorophyll (Canopy) & [43] \\
\hline $\begin{array}{l}\text { Transformed chlorophyll } \\
\text { absorption in reflectance } \\
\text { Index/Optimized soil-Adjusted } \\
\text { vegetation index }\end{array}$ & 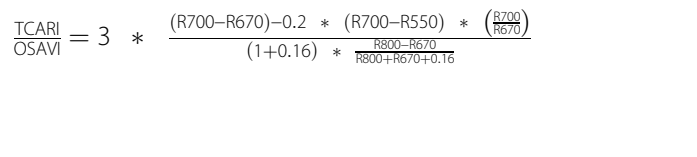 & Chlorophyll (Canopy) & [43] \\
\hline Triangular vegetation index & $\mathrm{TVI}=0.5 *(120 *(\mathrm{R} 750-\mathrm{R} 550)-200 *(\mathrm{R} 670-\mathrm{R} 550))$ & Leaf area and chlorophyll content (Canopy) & [44] \\
\hline Vogelmann & $V O G=\frac{R 740}{R 720}$ & Chlorophyll (Leaf) & [45] \\
\hline
\end{tabular}

The scale (leaf or canopy), at which these are commonly used, is also presented.

demostrated that HyperART measurements were consistent with data acquired with the well-established FluoWat device. We were able to show that the estimation of chlorophyll content of entire leaves, sampled from four different crops, was reasonably accurate. Moreover, it was shown that the system leads to an improved detection and quantification of disease symptoms (model plant-pathogen interaction Cercospora beticola and Beta vulgaris spp. vulgaris). Finally, it was noted that the non-absorbed radiation signal (i.e., $\rho+\tau$ ) reduced data noise in disease detection and significantly improved the estimation of leaf chlorophyll content across species with different leaf structure. Based on our results, we suggest that the HyperART system holds a high potential for studying plant responses to both biotic and abiotic stressors at the entire leaf level.

\section{Methods}

\section{Plant material and growth conditions}

Two experiments were conducted, each case study focused on another set of species. Both experiments took place in a greenhouse located in the western part of Germany (Jülich, $50^{\circ} \mathrm{N} ; 6^{\circ} \mathrm{E}$ ).

\section{Experiment I: Cercospora beticola infection}

Two single plants of a sugar beet line (Beta vulgaris ssp. vulgaris; identification number 8RF5006 (HS) KWS SAAT AG, Einbeck, Germany) were cultivated. During growth, air temperature and relative air humidity were $20.9 \pm 6.9^{\circ} \mathrm{C}$ (range: $8.5-47.6$ ) and $59.6 \pm 21.7 \%$ (range: $8.2-96.9$ ), respectively. Supplementary light was provided by highpressure sodium lamps (SON-T Agro, 250 W, Philips, 
Table 2 Vegetation indices performance based on a logarithmic regression model

\begin{tabular}{|c|c|c|c|c|c|c|c|c|c|c|c|c|c|}
\hline Index & Rank & Source & COR & $R^{2}$ & $F$ & $P$ & Index & Rank & Source & COR & $\mathrm{R}^{2}$ & $F$ & $\mathbf{P}$ \\
\hline \multirow[t]{3}{*}{$\overline{A N C B}$} & 8 & $\rho+\tau$ & 0.80 & 0.64 & 66.94 & 0.00 & PSNDb & 7 & $\tau$ & 0.80 & 0.64 & 67.86 & 0.00 \\
\hline & 13 & $\tau$ & 0.77 & 0.60 & 56.98 & 0.00 & & 20 & $\rho+\tau$ & 0.75 & 0.56 & 48.48 & 0.00 \\
\hline & 26 & $\rho$ & 0.71 & 0.50 & 37.60 & 0.00 & & 43 & $\rho$ & 0.55 & 0.30 & 16.15 & 0.00 \\
\hline \multirow[t]{3}{*}{ BGI2 } & 9 & $\rho$ & 0.80 & 0.63 & 65.65 & 0.00 & PSRI & 53 & $\tau$ & 0.37 & 0.14 & 6.02 & 0.02 \\
\hline & 27 & $\tau$ & -0.69 & 0.48 & 34.56 & 0.00 & & 54 & $\rho+\tau$ & 0.35 & 0.12 & 5.41 & 0.03 \\
\hline & 48 & $\rho+\tau$ & 0.46 & 0.22 & 10.41 & 0.00 & & 61 & $\rho$ & 0.15 & 0.02 & 0.82 & 0.37 \\
\hline \multirow[t]{3}{*}{ Chlg } & 38 & $\rho+\tau$ & 0.60 & 0.36 & 21.10 & 0.00 & PSSRa & 23 & $\tau$ & 0.74 & 0.55 & 45.88 & 0.00 \\
\hline & 41 & $\rho$ & 0.56 & 0.31 & 17.32 & 0.00 & & 49 & $\rho+\tau$ & 0.45 & 0.20 & 9.49 & 0.00 \\
\hline & 57 & $\tau$ & 0.25 & 0.06 & 2.58 & 0.12 & & 65 & $\rho$ & 0.08 & 0.01 & 0.23 & 0.64 \\
\hline \multirow[t]{3}{*}{ Chlre } & 15 & $\boldsymbol{\tau}$ & 0.77 & 0.59 & 54.90 & 0.00 & PSSRb & 12 & $\tau$ & 0.78 & 0.61 & 58.42 & 0.00 \\
\hline & 22 & $\rho+\tau$ & 0.75 & 0.56 & 47.42 & 0.00 & & 21 & $\rho+\tau$ & 0.75 & 0.56 & 48.36 & 0.00 \\
\hline & 33 & $\rho$ & 0.63 & 0.40 & 25.60 & 0.00 & & 51 & $\rho$ & 0.41 & 0.17 & 7.64 & 0.01 \\
\hline \multirow[t]{3}{*}{$\mathrm{EVI}$} & 1 & $\rho+\tau$ & 0.85 & 0.72 & 99.16 & 0.00 & SR1 & 10 & $\tau$ & 0.80 & 0.63 & 65.28 & 0.00 \\
\hline & 34 & $\tau$ & 0.62 & 0.38 & 23.21 & 0.00 & & 17 & $\rho+\tau$ & 0.76 & 0.58 & 53.06 & 0.00 \\
\hline & 44 & $\rho$ & 0.54 & 0.29 & 15.88 & 0.00 & & 31 & $\rho$ & 0.66 & 0.44 & 30.07 & 0.00 \\
\hline \multirow[t]{3}{*}{ MCARI } & 36 & $\rho$ & -0.61 & 0.37 & 22.07 & 0.00 & SIPI & 24 & $\tau$ & 0.72 & 0.52 & 41.39 & 0.00 \\
\hline & 46 & $\tau$ & 0.51 & 0.26 & 13.02 & 0.00 & & 52 & $\rho+\tau$ & 0.38 & 0.14 & 6.38 & 0.02 \\
\hline & 47 & $\rho+\tau$ & -0.49 & 0.24 & 12.24 & 0.00 & & 66 & $\rho$ & -0.03 & 0.00 & 0.03 & 0.86 \\
\hline \multirow[t]{3}{*}{$\mathrm{mND}$} & 3 & $\tau$ & 0.83 & 0.69 & 85.43 & 0.00 & SR2 & 18 & $\tau$ & 0.76 & 0.58 & 51.62 & 0.00 \\
\hline & 4 & $\rho+\tau$ & 0.82 & 0.67 & 76.13 & 0.00 & & 45 & $\rho+\tau$ & 0.53 & 0.29 & 15.16 & 0.00 \\
\hline & 28 & $\rho$ & 0.69 & 0.47 & 34.36 & 0.00 & & 63 & $\rho$ & 0.10 & 0.01 & 0.42 & 0.52 \\
\hline \multirow[t]{3}{*}{$\mathrm{mSR}$} & 14 & $\tau$ & 0.77 & 0.60 & 56.46 & 0.00 & TCARI & 37 & $\rho$ & -0.60 & 0.36 & 21.16 & 0.00 \\
\hline & 40 & $\rho+\tau$ & -0.57 & 0.32 & 18.27 & 0.00 & & 42 & $\rho+\tau$ & -0.56 & 0.31 & 17.09 & 0.00 \\
\hline & 56 & $\rho$ & -0.29 & 0.08 & 3.46 & 0.07 & & 64 & $\tau$ & 0.09 & 0.01 & 0.32 & 0.57 \\
\hline \multirow[t]{3}{*}{ ND } & 2 & $\boldsymbol{\tau}$ & 0.84 & 0.70 & 90.52 & 0.00 & TCARI/OSAVI & 30 & $\rho$ & -0.67 & 0.44 & 30.36 & 0.00 \\
\hline & 6 & $\rho+\tau$ & 0.80 & 0.65 & 69.52 & 0.00 & & 32 & $\rho+\tau$ & -0.66 & 0.43 & 29.11 & 0.00 \\
\hline & 29 & $\rho$ & 0.69 & 0.47 & 34.36 & 0.00 & & 59 & $\tau$ & -0.21 & 0.04 & 1.71 & 0.20 \\
\hline \multirow[t]{3}{*}{ NDVI } & 16 & $\tau$ & 0.77 & 0.59 & 54.01 & 0.00 & $\mathrm{TVl}$ & 50 & $\rho+\tau$ & 0.43 & 0.19 & 8.68 & 0.01 \\
\hline & 35 & $\rho+\tau$ & 0.62 & 0.38 & 23.20 & 0.00 & & 55 & $\tau$ & 0.29 & 0.09 & 3.57 & 0.07 \\
\hline & 58 & $\rho$ & 0.23 & 0.05 & 2.16 & 0.15 & & 62 & $\rho$ & 0.14 & 0.02 & 0.81 & 0.37 \\
\hline \multirow[t]{3}{*}{ PSNDa } & 19 & $\tau$ & 0.76 & 0.57 & 50.71 & 0.00 & VOG & 5 & $\tau$ & 0.82 & 0.66 & 75.39 & 0.00 \\
\hline & 39 & $\rho+\tau$ & 0.57 & 0.33 & 18.40 & 0.00 & & 11 & $\rho+\tau$ & 0.78 & 0.61 & 60.69 & 0.00 \\
\hline & 60 & $\rho$ & 0.18 & 0.03 & 1.22 & 0.28 & & 25 & $\rho$ & 0.71 & 0.50 & 37.91 & 0.00 \\
\hline
\end{tabular}

Determination $\left(\mathrm{R}^{2}\right)$ and correlation (COR) coefficients, as well as significance level of the relation between the 22 vegetation indices (abbreviations in Table 1) and destructively-measured chlorophyll content. Vegetation indices were calculated based on reflectance $(\rho)$, transmittance $(\tau)$ or combination of $\rho$ with $\tau$ (i.e., $\rho++\tau$ ) source images. Indices were ranked based on the $R^{2}$ value (all three source images were included in the ranking). The entire leaf surface was averaged ( $=40$ ). All four species (maize, rapeseed, barley and tomato) were plotted together (examples are shown in Additional file 1: Figure S1). Plants were grown under control or deficient nitrogen levels. Bold text indicates $\mathrm{R}^{2}$ values greater or equal to 0.5 .

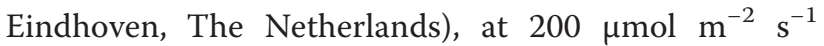
photosynthetic photon flux density for $16 \mathrm{~h}$ per day. Plants were grown in $1 \mathrm{~L}$ pots, filled with soil mixture $(1: 1, \mathrm{v} / \mathrm{v})$ of Hawita Dachstaudensubstrat (HAWITA GRUPPE GmbH, Vechta, Germany) and Pikiererde (Einheitserde Typ P Pikier, Balster Einheitserdewerk $\mathrm{GmbH}$, Frödenberg, Germany). Plants were kept wellwatered, and were weekly provided with nutrient solution (0.2\%; Hakaphos Blau, COMPO GmbH \& Co. KG,
Münster, Germany) starting from the second week following emergence. The amount of nutrient solution was $60 \mathrm{~mL}$ per plant (up to 4 weeks following emergence), and was subsequently increased to $80 \mathrm{~mL}$. Plants were grown for six weeks (starting from $17^{\text {th }}$ of July, 2012) up to the developmental stage 18 of the $\mathrm{BBCH}$ scale [52].

Before inoculation, the pathogen Cercospora beticola was grown on agar plates (1.5\%; Sigma-Aldrich Chemie $\mathrm{GmbH}$, Taufkirchen, Germany), containing 50\% vegetable 
Table 3 Vegetation indices performance based on a linear regression model

\begin{tabular}{|c|c|c|c|c|c|c|c|c|c|c|c|c|c|}
\hline Index & Rank & Source & COR & $R^{2}$ & $F$ & $P$ & Index & Rank & Source & COR & $\mathrm{R}^{2}$ & $F$ & $\mathbf{P}$ \\
\hline \multirow[t]{3}{*}{$\overline{A N C B}$} & 12 & $\tau$ & 0.75 & 0.56 & 49.14 & 0.00 & PSNDb & 17 & $\tau$ & 0.73 & 0.53 & 42.17 & 0.00 \\
\hline & 15 & $\rho+\tau$ & 0.74 & 0.55 & 45.75 & 0.00 & & 23 & $\rho+\tau$ & 0.65 & 0.43 & 28.49 & 0.00 \\
\hline & 28 & $\rho$ & 0.62 & 0.39 & 24.20 & 0.00 & & 49 & $\rho$ & 0.45 & 0.20 & 9.53 & 0.00 \\
\hline \multirow[t]{3}{*}{ BGI2 } & 5 & $\rho$ & 0.78 & 0.61 & 59.78 & 0.00 & PSRI & 52 & $\tau$ & 0.34 & 0.12 & 4.97 & 0.03 \\
\hline & 31 & $\tau$ & -0.60 & 0.36 & 21.79 & 0.00 & & 56 & $\rho+\tau$ & 0.27 & 0.07 & 3.05 & 0.09 \\
\hline & 42 & $\rho+\tau$ & 0.50 & 0.25 & 12.68 & 0.00 & & 64 & $\rho$ & 0.05 & 0.00 & 0.09 & 0.77 \\
\hline \multirow[t]{3}{*}{ Chlg } & 37 & $\rho+\tau$ & 0.55 & 0.30 & 16.15 & 0.00 & PSSRa & 18 & $\tau$ & 0.71 & 0.51 & 39.52 & 0.00 \\
\hline & 43 & $\rho$ & 0.49 & 0.24 & 11.86 & 0.00 & & 50 & $\rho+\tau$ & 0.37 & 0.14 & 6.16 & 0.02 \\
\hline & 57 & $\tau$ & 0.23 & 0.05 & 2.12 & 0.15 & & 66 & $\rho$ & 0.02 & 0.00 & 0.02 & 0.88 \\
\hline \multirow[t]{3}{*}{ Chlre } & 13 & $\boldsymbol{\tau}$ & 0.75 & 0.56 & 47.68 & 0.00 & PSSRb & 9 & $\tau$ & 0.76 & 0.58 & 52.50 & 0.00 \\
\hline & 19 & $\rho+\tau$ & 0.71 & 0.50 & 38.59 & 0.00 & & 20 & $\rho+\tau$ & 0.70 & 0.48 & 35.76 & 0.00 \\
\hline & 34 & $\rho$ & 0.59 & 0.34 & 19.80 & 0.00 & & 53 & $\rho$ & 0.33 & 0.11 & 4.67 & 0.04 \\
\hline \multirow[t]{3}{*}{ EVI } & 1 & $\rho+\tau$ & 0.83 & 0.70 & 87.37 & 0.00 & SR1 & 6 & $\tau$ & 0.78 & 0.60 & 57.58 & 0.00 \\
\hline & 24 & $\tau$ & 0.65 & 0.42 & 27.30 & 0.00 & & 16 & $\rho+\tau$ & 0.73 & 0.54 & 43.81 & 0.00 \\
\hline & 40 & $\rho$ & 0.52 & 0.27 & 13.72 & 0.00 & & 29 & $\rho$ & 0.62 & 0.39 & 23.97 & 0.00 \\
\hline \multirow[t]{3}{*}{ MCARI } & 35 & $\rho$ & -0.58 & 0.34 & 19.23 & 0.00 & SIPI & 30 & $\tau$ & 0.61 & 0.37 & 22.38 & 0.00 \\
\hline & 39 & $\rho+\tau$ & -0.52 & 0.27 & 14.32 & 0.00 & & 55 & $\rho+\tau$ & 0.29 & 0.08 & 3.39 & 0.07 \\
\hline & 48 & $T$ & 0.46 & 0.21 & 10.01 & 0.00 & & 63 & $\rho$ & -0.08 & 0.01 & 0.27 & 0.60 \\
\hline \multirow[t]{3}{*}{$\mathrm{mND}$} & 4 & $\tau$ & 0.80 & 0.64 & 67.83 & 0.00 & SR2 & 14 & $\tau$ & 0.74 & 0.55 & 45.79 & 0.00 \\
\hline & 7 & $\rho+\tau$ & 0.77 & 0.59 & 55.58 & 0.00 & & 47 & $\rho+\tau$ & 0.46 & 0.21 & 10.05 & 0.00 \\
\hline & 26 & $\rho$ & 0.63 & 0.39 & 24.65 & 0.00 & & 65 & $\rho$ & 0.04 & 0.00 & 0.06 & 0.81 \\
\hline \multirow[t]{3}{*}{$\mathrm{mSR}$} & 10 & $\tau$ & 0.75 & 0.57 & 49.85 & 0.00 & TCARI & 38 & $\rho$ & -0.54 & 0.29 & 15.61 & 0.00 \\
\hline & 33 & $\rho+\tau$ & -0.59 & 0.35 & 20.20 & 0.00 & & 45 & $\rho+\tau$ & -0.48 & 0.23 & 11.14 & 0.00 \\
\hline & 54 & $\rho$ & -0.31 & 0.10 & 4.15 & 0.05 & & 60 & $\tau$ & 0.13 & 0.02 & 0.67 & 0.42 \\
\hline \multirow[t]{3}{*}{ ND } & 3 & $\boldsymbol{\tau}$ & 0.80 & 0.64 & 68.15 & 0.00 & TCARI/OSAVI & 32 & $\rho$ & -0.59 & 0.35 & 20.27 & 0.00 \\
\hline & 11 & $\rho+\tau$ & 0.75 & 0.56 & 49.16 & 0.00 & & 36 & $\rho+\tau$ & -0.56 & 0.31 & 17.12 & 0.00 \\
\hline & 27 & $\rho$ & 0.63 & 0.39 & 24.65 & 0.00 & & 59 & $\tau$ & -0.13 & 0.02 & 0.68 & 0.41 \\
\hline \multirow[t]{3}{*}{ NDVI } & 22 & $\tau$ & 0.66 & 0.43 & 28.78 & 0.00 & $\mathrm{TVl}$ & 44 & $\rho+\tau$ & 0.48 & 0.23 & 11.46 & 0.00 \\
\hline & 41 & $\rho+\tau$ & 0.51 & 0.26 & 13.30 & 0.00 & & 51 & $\tau$ & 0.35 & 0.12 & 5.35 & 0.03 \\
\hline & 58 & $\rho$ & 0.16 & 0.03 & 1.00 & 0.32 & & 61 & $\rho$ & 0.13 & 0.02 & 0.67 & 0.42 \\
\hline \multirow[t]{3}{*}{ PSNDa } & 25 & $\tau$ & 0.64 & 0.41 & 26.88 & 0.00 & VOG & 2 & $\tau$ & 0.80 & 0.64 & 68.85 & 0.00 \\
\hline & 46 & $\rho+\tau$ & 0.47 & 0.22 & 10.87 & 0.00 & & 8 & $\rho+\tau$ & 0.76 & 0.58 & 52.70 & 0.00 \\
\hline & 62 & $\rho$ & 0.12 & 0.01 & 0.56 & 0.46 & & 21 & $\rho$ & 0.68 & 0.46 & 32.31 & 0.00 \\
\hline
\end{tabular}

Determination $\left(\mathrm{R}^{2}\right)$ and correlation (COR) coefficients, as well as significance level of the relation between the 22 vegetation indices (abbreviations in Table 1) and destructively-measured chlorophyll content. Vegetation indices were calculated based on reflectance $(\rho)$, transmittance $(\tau)$ or combination of $\rho$ with $\tau$ (i.e., $\rho++\tau$ ) source images. Indices were ranked based on the $R^{2}$ value (all three source images were included in the ranking). The entire leaf surface was averaged ( $n=40$ ). All four species (maize, rapeseed, barley and tomato) were plotted together. Plants were grown under control or deficient nitrogen levels. Bold text indicates $\mathrm{R}^{2}$ values greater or equal to 0.5 .

juice (Gemüsemix, Eckes-Granini Deutschland GmbH, Nieder-Olm, Germany). Pathogen cultivation took place in a growth chamber, set at $60 \%$ relative air humidity and $26^{\circ} \mathrm{C}$ air temperature. The pathogen was kept on the agar plates for 3 weeks. To stimulate conidia production, plates were placed under ultraviolet light $(340-400 \mathrm{~nm})$ for 3 days before conidia collection. Subsequently, conidia were scraped with a slide, and were suspended to an aqueous solution containing Tween20 (0.1\%; Sigma-Aldrich
Chemie GmbH, Taufkirchen, Germany). The solution concentration was set to $3 \times 10^{4}$ conidia $\mathrm{mL}^{-1}$ using a hemocytometer (Thoma chamber, Carl Roth $\mathrm{GmbH}+\mathrm{Co}$. KG, Karlsruhe, Germany). Plant inoculation was conducted, as previously described by Schmidt et al. (2008) [53]. Following inoculation, plants were kept at $80-100 \%$ relative air humidity for one week. During that time, air temperature was $24 \pm 0.3^{\circ} \mathrm{C}$ (range: $15-35$ ). Light intensity was set to $300 \mu \mathrm{mol} \mathrm{m} \mathrm{m}^{-2} \mathrm{~s}^{-1}$ one day after inoculation. 
The control plants were inoculated with an aqueous solution containing only Tween 20 (0.1\%). Measurements were conducted about three weeks after inoculation, when symptoms of Cercospora beticola infection were visible.

\section{Experiment II: Nitrogen deficit}

Two dicotyledonous crops [rapeseed (Brassica rapa cv. Campino) and tomato (Lycopersicon esculentum cv. Harzfeuer)] and two monocotyledonous crops [maize (Zea mays cv. Gelber) and barley (Hordeum vulgare cv. Barke)] were grown under controlled conditions in a greenhouse. During growth period, air temperature and relative air humidity were $21.1 \pm 0.3^{\circ} \mathrm{C}$ (range: 19.7-23.9) and $51 \pm 1 \%$ (range: 43-60), respectively. Supplementary light was provided by high-pressure sodium lamps, as described above, at $50 \mu \mathrm{mol} \mathrm{m} \mathrm{m}^{-2}$ photosynthetic photon flux density for $16 \mathrm{~h}$ per day (from 0600 to 2200 hours). The average daily light integral, including natural light, was $9.6 \pm 0.5 \mathrm{~mol} \mathrm{~m}{ }^{-2}$ day $^{-1}$. Seeds were sown and germinated in small pots until plants were grown up to the cotyledons' stage (i.e. fully open cotyledons and before the appearance of the first leaf for dicotyledonous species and the second leaf stage for monocotyledonous species). Subsequently, seedlings were transplanted into 17.4 L rectangular boxes containing a ready-made soil mixture ('Nullerde' Archut Erzeugnisse GmbH, Vechta, Germany) with low nitrogen content $\left(50 \mathrm{mg} \mathrm{L}^{-1}\right)$. A planting density of five plants per box was used. Boxes were randomly distributed on a glasshouse table.

After planting, plants were exposed to different levels of nitrogen fertilisation. For this, eight boxes, containing five plants each, were separated into two different treatments: control (10 $\mathrm{mM}$ nitrogen concentration) and nitrogen deficit (15\% of the control). Once a week, each box received $500 \mathrm{~mL}$ of nutrient solution [54]. The experiment lasted for 23 days, following planting (starting from 25th of April, 2013).

\section{Point measurements}

The spectral signature of selected leaves was also obtained by using a high spectral resolution point spectroradiometer (ASD FieldSpec ${ }^{\bullet}$ 3, Analytical Spectral Devices, Boulder, CO, USA), operating at a spectral range between 350 and $2500 \mathrm{~nm}$. The nominal spectral resolution is 3 and $10 \mathrm{~nm}$ FWHM in the 350-1050 and 1050-2500 nm regions, respectively. The sampling interval is 1.4 and $2 \mathrm{~nm}$ in the 350-1050 and 1050-2500 nm regions, respectively. The FieldSpec fibre optic was connected to a FluoWat leaf clip $[17,18]$. The FluoWat leaf clip offers the possibility to measure both reflectance and transmittance of either sample side. Illumination was provided by a cold light source (Schott KL 1500 LCD 150 W, Lighting and Imaging SCHOTT AG, Mainz, Germany). Due to the spectral output of the illumination source, spectral readings were limited to the 400 to $1700 \mathrm{~nm}$ range. The intensity of the electromagnetic radiation emission was determined by using a $99.9 \%$ reflective white standard (Spectralon, Labsphere Inc., North Dutton, NH, USA). The spectra of the samples were divided by the white standard spectra and multiplied by a correction factor (provided by Labsphere Inc.) for calculation of the relative values. In all measurements, leaf reflectance was assessed on the adaxial (upper) surface.

\section{Spectra comparison}

To assess the validity of the spectral data acquired using the HyperART system (first prototype), simultaneous measurements using both this and the FluoWat device were performed on three disease-free sugar beet leaves from the control plant. Five FluoWat point measurements were averaged, and these were tested against five comparable points that were selected in the hypespectral image obtained by using the HyperART system. In this study we combine the spectra of two cameras by selecting nearly the same regions in both hyperspectral images and averaging them. Since the investigated plant tissue was homogeneous, and thus signal changes spatially smooth, we assume that the spectral differences do not change much when the regions of interest do not fit perfectly.

The sensors of these two compared instruments have similar spectral resolution, whereas spectral sampling differs. For comparison of the spectra obtained by the two instruments, the data were converted to a similar spectral sampling. This was done by reducing the FieldSpec spectral sampling to the one of the line scanners, by matching the wavelength.

\section{Quantification of Cercospora beticola symptoms}

The potential of the HyperART system in detecting and quantifying the symptoms of Cercospora beticola infection, on sugar beet leaves was evaluated. Three leaves of an infected plant and three leaves of an infection-free plant, were placed in the leaf clip between the mirrors of the HyperART system (first prototype). Two leaves of the infected plant had necrotic areas, whereas the third one did not have any disease symptoms. Disease severity was also estimated visually (expressed in percentage) for each leaf.

The background of the hyperspectral image was masked out using a threshold in the EVI image. After image processing, a disease specific index (Cercospora leaf spot index $\left.(C L S I)=\frac{\rho 698-\rho 750}{\rho 698+\rho 750}-\rho 734 ;[13]\right)$ was calculated from the $\rho, \tau$ and $\rho+\tau$ data, respectively. Low values indicate low disease severity, and vice-versa. From the CLSI images, histograms with a binning of 0.01 were computed and divided by overall number of pixels. By this the normalized histograms represent estimated probability density function of CLSI values. As an indicator of 
how well the two modes can be distinguished, we calculated the so-called bimodal amplitude (referred as $A_{\mathrm{b}}$ ). The bimodal amplitude equation $\left(A_{b}=\frac{A_{m}-A_{v}}{A_{m}} ;[55]\right)$ was used to analyse the CLSI histograms. For calculating $A_{b}$, the smaller peak amplitude (referred as $\mathrm{A}_{\mathrm{m}}$ ) and the amplitude of the minimum between the two peaks (referred as $A_{v}$ ) were computed. Peak heights (normalized frequencies) and locations were 0.021 at $\rho$ CLSI -1.21 value, 0.022 at TCLSI -1.32 value and 0.014 at $\rho+$ TCLSI -1.42 value. The minima normalized frequencies were 0.005 at -0.75 value, 0.004 at -0.75 value, and 0.004 at -0.75 value for $\rho C L S I, \tau C L S I$ and $\rho+\tau C L S I$, respectively. $A_{b}$ values close to one indicate a better double peak distinction.

In order to evaluate the separability performance in the index images of $\rho$ CLSI, $\tau$ CLSI and $\rho+\tau$ CLSI, these were firstly classified by using the K-Means unsupervised classification (ENVI 4.8; [56]). The automatic algorithm was set to separate the data in the following two classes: infected and non-infected leaf tissue. The same classes were used for a supervised classification performed by support vector machine on the $\rho, \tau$ and $\rho+\tau$ hyperspectral images. Therefore, symptomatic and symptomfree regions were selected manually in the hyperspectral images. After all images were classified, the validity of unsupervised classification performance was evaluated using post classification. Unsupervised classification results for the index images of $\rho$ CLSI, $\tau$ CLSI and $\rho+\tau C L S I$ were compared by confusion matrix with the respective supervised classification (ENVI 4.8). The supervised classification results were used as ground true images.

\section{Vegetation indices}

Twenty two commonly-used indices for chlorophyll content estimation at both leaf and canopy levels (Table 1) were applied to $\rho, \tau$, and $\rho+\tau$ pre-processed hyperspectral images. Throughout the paper, the letters $\rho$, $\tau$, and $\rho+\tau$ before an index indicate the source of the hyperspectral image, which was used for the calculation. Sixty six outcomes (i.e., 22 indices $\times 3$ sources) were obtained. Both a simple linear regression and a logarithmic model were applied to the relationships between predicted (by the indices) and destructively-measured (described below) chlorophyll content.

\section{Assessment of chlorophyll content}

Sampling for biochemical assessment of chlorophyll content was conducted 23 days after planting. The third and fourth (counting from the apex) fully-expanded leaves were collected for rapeseed, barley and tomato (two leaves per plant; $\mathrm{n}=10$ ). The middle portion of the third fullyexpanded was sampled for maize (one leaf per plant; $n=5$ ). Chlorophyll concentration was assessed in the same leaves, where hyperspectral measurements were done.
Non-destructive (hyperspectral imaging) and destructive (chlorophyll content) measurements were performed within $24 \mathrm{~h}$.

Collected plant material was immediately frozen in liquid nitrogen, and stored at $-80^{\circ} \mathrm{C}$ for further analysis. Plant material was homogenized by grinding the tissue in liquid nitrogen. About $50 \mathrm{mg}$ of the homogenized sample were ground in $6 \mathrm{ml}$ acetone (100\% pure solvent), by using a mortar and pestle. Since chlorophyll is light sensitive, extraction took place in a dark room. Liquid nitrogen was continuously used to cool down the sample. The resulting homogenate was centrifuged in test tubes for $6 \mathrm{~min}$ for precipitation of the cell debris. Absorption spectra of the supernatants were recorded. Chlorophyll content was calculated by equations described by Lichtenthaler [57].

\section{Additional file}

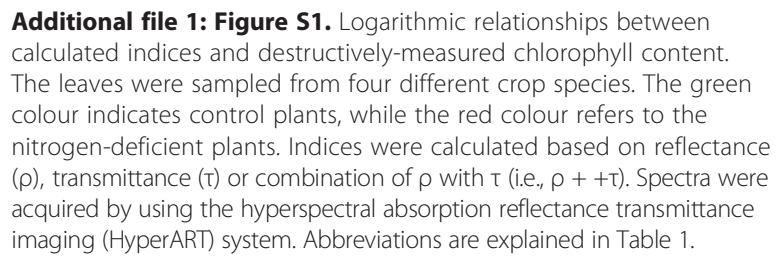

\section{Abbreviations}

a: Absorption; CLSI: Cercospora leaf spot index; $A_{b}$ : Bimodal amplitude; HyperART: Hyperspectral absorption-reflectance-transmittance imaging; NIR: Near infrared; PAR: Photosynthetic active radiation; $\rho$ : Reflectance; $\tau$ : Transmittance.

\section{Competing interests}

The authors declare that they have no competing interests.

\section{Authors' contributions}

SB developed the HyperART system, acquired the spectral data, carried out the data analysis and interpretation, and wrote the manuscript. DF carried out the second case study, and contributed to the writing of the paper. SS was responsible for the biotic stress handling (first case study) and supported the respective measurements. MPC assisted the data analysis and interpretation. MJ, HS and UR designed experiments, participated in data interpretation and supervised the study. All authors contributed in writing, editing, proof-reading and approving the final manuscript.

\section{Acknowledgements}

This work has been conducted in frame of a research program funded by the BMBF with the project number 315309/CROP.SENSe and by the

European Regional Development Fund [ERDF (En), EFRE (De)]. It was partly funded by the European Plant Phenotyping Network (EPPN -European Union FP7 Capacities Programme) through a post-doctoral grant to DF (grant agreement 284443). We thank the company KWS SAAT AG for providing the plant and pathogen material of the first case study. We are grateful to Hans Jung and the FZJ IBG-workshop/ZEA-1 personnel for assembling the prototypes. Thanks also to Dr. Mark Müller-Linow and Dr. Zbyněk Malenovský for providing the MatLab script. The authors are thankful to Anna Krapp for assistance in the preparation of figures and tables. Insightful discussions with Dr. Fabio Fiorani are greatly appreciated. Finally, we thank the editor and two anonymous reviewers for their valuable comments on the manuscript.

\section{Author details}

${ }^{1}$ Institute for Bio- and Geosciences, IBG-2: Plant Sciences, Forschungszentrum Jülich $\mathrm{GmbH}, 52425$ Jülich, Germany. ${ }^{2}$ Present address: Department of Crop Science, Technological Educational Institute of Crete, GR 71004 Heraklio, 
Greece. ${ }^{3}$ Present address: Institute of Viticulture, Floriculture and Vegetable Crops, Hellenic Agricultural Organization 'Demeter' (NAGREF), P.O. Box 2228, GR 71003 Heraklio, Greece. ${ }^{4}$ Present address: LemnaTec GmbH, Pascalstraße 59, 52076 Aachen, Germany.

\section{Received: 11 October 2014 Accepted: 3 January 2015} Published online: 16 January 2015

\section{References}

1. Jansen M, Pinto F, Nagel KA, Dusschoten D, Fiorani F, Rascher U, et al. Non-invasive phenotyping methodologies enable the accurate characterization of growth and performance of shoots and roots. In: Tuberosa R, Graner A,

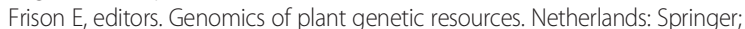
2014. p. 173-206.

2. Fiorani F, Schurr U. Future scenarios for plant phenotyping. Annu Rev Plant Biol. 2013;64:267-91.

3. Dhondt S, Wuyts N, Inzé D. Cell to whole-plant phenotyping: the best is yet to come. Trends Plant Sci. 2013;18:428-39.

4. Furbank RT, Tester M. Phenomics - technologies to relieve the phenotyping bottleneck. Trends Plant Sci. 2011;16:635-44.

5. Fanourakis D, Briese C, Max J, Kleinen S, Putz A, Fiorani F, et al. Rapid determination of leaf area and plant height by using light curtain arrays in four species with contrasting shoot architecture. Plant Methods. 2014;10:9.

6. Fiorani F, Rascher U, Jahnke S, Schurr U. Imaging plants dynamics in heterogenic environments. Curr Opin Biotechnol. 2012;23:227-35.

7. Rascher U, Blossfeld S, Fiorani F, Jahnke $S$, Jansen M, Kuhn AJ, et al. Non-invasive approaches for phenotyping of enhanced performance traits in bean. Funct Plant Biol. 2011;38:968-83.

8. Matsuda O, Tanaka A, Fujita T, Iba K. Hyperspectral imaging techniques for rapid identification of arabidopsis mutants with altered leaf pigment status. Plant Cell Physiol. 2012;53:1154-70.

9. Rumpf T, Romer C, Plumer L, Mahlein AK, leee. Optimal wavelengths for an early identification of cercospora beticola with support vector machines based on hyperspectral reflection data. New York: leee; 2010.

10. Rumpf T, Mahlein AK, Steiner U, Oerke EC, Dehne HW, Plümer L. Early detection and classification of plant diseases with support vector machines based on hyperspectral reflectance. Comput Electron Agr. 2010;74:91-9.

11. Mahlein AK, Steiner U, Dehne HW, Oerke EC. Spectral signatures of sugar beet leaves for the detection and differentiation of diseases. Precis Agr. 2010;11:413-31.

12. Mahlein AK, Steiner U, Hillnhütter C, Dehne HW, Oerke EC. Hyperspectral imaging for small-scale analysis of symptoms caused by different sugar beet diseases. Plant Methods. 2012:8:3.

13. Mahlein AK, Rumpf T, Welke $P$, Dehne HW, Plümer L, Steiner $U$, et al. Development of spectral indices for detecting and identifying plant diseases. Remote Sens Environ. 2013;128:21-30.

14. Van Wittenberghe S, Verrelst J, Rivera JP, Alonso L, Moreno J, Samson R. Gaussian processes retrieval of leaf parameters from a multi-species reflectance, absorbance and fluorescence dataset. J Photochem Photobiol B Biol. 2014;134:37-48.

15. Gitelson AA, Buschmann C, Lichtenthaler HK. Leaf chlorophyll fluorescence corrected for re-absorption by means of absorption and reflectance measurements. J Plant Physiol. 1998;152:283-96.

16. Lukes $P$, Stenberg $P$, Rautiainen $M$, Mottus $M$, Vanhatalo $K$, Lukeš $P$, et al. Optical properties of leaves and needles for boreal tree species in Europe. Rem Sens Lett. 2013:4:667-76.

17. Alonso L, Gomez-Chova L, Vila-Frances J, Amoros-Lopez J, Guanter L, Calpe J, et al. Sensitivity analysis of the fraunhofer line discrimination method for the measurement of chlorophyll fluorescence using a field spectroradiometer. 2007. 7-9 February edition. Florence, Italy: Proceedings of the 3th International Workshop on Remote Sensing of Vegetation Fluorescence.

18. Van Wittenberghe S, Alonso L, Verrelst J, Hermans I, Delegido J, Veroustraete F, et al. Upward and downward solar-induced chlorophyll fluorescence yield indices of four tree species as indicators of traffic pollution in Valencia. Environ Pollut. 2013;173:29-37.

19. Vedaldi A, Fulkerson B. VLFeat: an open and portable library of computer vision algorithms. In: Proceedings of The International Conference on Multimedia. Firenze, Italy: ACM; 2010. p. 1469-72.

20. Eller BM. Leaf pubescence - significance of lower surface hairs for spectral properties of upper surface. J Exp Bot. 1977;28:1054-9.
21. Kokaly RF, Asner GP, Ollinger SV, Martin ME, Wessman CA. Characterizing canopy biochemistry from imaging spectroscopy and its application to ecosystem studies. Remote Sens Environ. 2009;113(Supplement 1):S78-91.

22. Delegido J, Verrelst J, Rivera JP, Ruiz-Verdú A, Moreno J. Brown and green LAl mapping through spectral indices. Int J Appl Earth Observation Geoinform. 2015;35:350-8.

23. Jansen M, Bergsträsser S, Schmittgen S, Müller-Linow M, Rascher U. Non-invasive spectral phenotyping methods can improve and accelerate cercospora disease scoring in sugar beet breeding. Agriculture. 2014;4:147-58.

24. Buschmann C, Lenk S, Lichtenthaler HK. Reflectance spectra and images of green leaves with different tissue structure and chlorophyll content. Israel J Plant Sci. 2012;60:49-64.

25. Horler DNH, Dockray M, Barber J. The red edge of plant leaf reflectance. Int J Rem Sens. 1983:4:273-88.

26. Gitelson AA, Gritz Y, Merzlyak MN. Relationships between leaf chlorophyll content and spectral reflectance and algorithms for non-destructive chlorophyll assessment in higher plant leaves. J Plant Physiol. 2003;160:271-82.

27. Filella I, Penuelas J. The red edge position and shape as indicators of plant chlorophyll content, biomass and hydric status. Int J Rem Sens. 1994;15:1459-70.

28. Thomas JR, Gausman HW. Leaf reflectance vs. leaf chlorophyll and carotenoid concentrations for eight crops. Agron J. 1977;69:799-802.

29. Bock CH, Poole GH, Parker PE, Gottwald TR. Plant disease severity estimated visually, by digital photography and image analysis, and by hyperspectral imaging. Crit Rev Plant Sci. 2010;29:59-107.

30. Malenovský Z, Homolová L, Zurita-Milla R, Lukeš P, Kaplan V, Hanuš J, et al. Retrieval of spruce leaf chlorophyll content from airborne image data using continuum removal and radiative transfer. Remote Sens Environ. 2013;131:85-102.

31. Zarco-Tejada PJ, Berjón A, López-Lozano R, Miller JR, Martín P, Cachorro V, et al. Assessing vineyard condition with hyperspectral indices: leaf and canopy reflectance simulation in a row-structured discontinuous canopy. Remote Sens Environ. 2005;99:271-87.

32. Gitelson AA, Keydan GP, Merzlyak MN. Three-band model for noninvasive estimation of chlorophyll, carotenoids, and anthocyanin contents in higher plant leaves. Geophys Res Lett. 2006;33:L11402.

33. Huete AR, Liu HQ, Batchily K, van Leeuwen W. A comparison of vegetation indices over a global set of TM images for EOS-MODIS. Remote Sens Environ. 1997:59:440-51.

34. Daughtry CST, Walthall CL, Kim MS, de Colstoun EB, McMurtrey III JE. Estimating corn leaf chlorophyll concentration from leaf and canopy reflectance. Remote Sens Environ. 2000;74:229-39.

35. Sims DA, Gamon JA. Relationships between leaf pigment content and spectral reflectance across a wide range of species, leaf structures and developmental stages. Remote Sens Environ. 2002;81:337-54.

36. Gitelson A, Merzlyak MN. Quantitative estimation of chlorophyll-a using reflectance spectra: Experiments with autumn chestnut and maple leaves. J Photochem Photobiol B Biol. 1994;22:247-52.

37. Rouse JW, Haas RH, Schell JA, Deering DW. Monitoring Vegetation Systems in the Great Plains with ERTS, Third ERTS Symposium, NASA SP-351. 1973. p. 309-17.

38. Penuelas J, Baret F, Filella I. Semiempirical indexes to assess carotenoids chlorophyll-a ration from leaf spectral reflectance. Photosynthetica. 1995;31:221-30.

39. Zarco-Tejada PJ, Miller JR, Noland TL, Mohammed GH, Sampson PH. Scaling-up and model inversion methods with narrowband optical indices for chlorophyll content estimation in closed forest canopies with hyperspectral data. Geosci Rem Sens IEEE Trans On. 2001;39:1491-507.

40. Birth GS, McVey GR. Measuring color of growing turf with a reflectance spectrophotometer. Agron J. 1968;60:640-9.

41. Blackburn GA. Spectral indices for estimating photosynthetic pigment concentrations: a test using senescent tree leaves. Int J Rem Sens. 1998;19:657-75.

42. Merzlyak MN, Gitelson AA, Chivkunova OB, Rakitin WYU. Non-destructive optical detection of pigment changes during leaf senescence and fruit ripening. Physiol Plant. 1999;106:135-41.

43. Haboudane D, Miller JR, Tremblay N, Zarco-Tejada PJ, Dextraze L. Integrated narrow-band vegetation indices for prediction of crop chlorophyll content for application to precision agriculture. Remote Sens Environ. 2002;81:416-26.

44. Broge NH, Leblanc E. Comparing prediction power and stability of broadband and hyperspectral vegetation indices for estimation of green leaf area index and canopy chlorophyll density. Remote Sens Environ. 2001;76:156-72. 
45. Vogelmann JE, Rock BN, Moss DM. Red edge spectral measurements from sugar maple leaves. Int J Rem Sens. 1993;14:1563-75.

46. Buschmann C, Nagel E. In vivo spectroscopy and internal optics of leaves as basis for remote sensing of vegetation. Int J Rem Sens. 1993;14:711-22.

47. Main R, Cho MA, Mathieu R, O'Kennedy MM, Ramoelo A, Koch S. An investigation into robust spectral indices for leaf chlorophyll estimation. ISPRS J Photogram Rem Sens. 2011;66:751-61.

48. le Maire G, François C, Dufrêne E. Towards universal broad leaf chlorophyll indices using PROSPECT simulated database and hyperspectral reflectance measurements. Remote Sens Environ. 2004;89:1-28.

49. Asner GP, Martin RE. Spectral and chemical analysis of tropical forests: scaling from leaf to canopy levels. Remote Sens Environ. 2008;112:3958-70.

50. Delegido J, Alonso L, González G, Moreno J. Estimating chlorophyll content of crops from hyperspectral data using a normalized area over reflectance curve (NAOC). Int J Appl Earth Observation Geoinform. 2010;12:165-74.

51. Ji-Yong S, Xiao-Bo Z, Jie-Wen Z, Kai-Liang W, Zheng-Wei C, Xiao-Wei H, et al. Nondestructive diagnostics of nitrogen deficiency by cucumber leaf chlorophyll distribution map based on near infrared hyperspectral imaging. Sci Hortic. 2012;138:190-7.

52. Hess M, Barralis G, Bleiholder H, Buhr L, Eggers TH, Hack H, et al. Use of the extended $\mathrm{BBCH}$ scale - general for the descriptions of the growth stages of mono; and dicotyledonous weed species. Weed Res. 1997;37:433-41.

53. Schmidt K, Pflugmacher M, Klages S, Maser A, Mock A, Stahl DJ. Accumulation of the hormone abscisic acid (ABA) at the infection site of the fungus Cercospora beticola supports the role of $A B A$ as a repressor of plant defence in sugar beet. Mol Plant Pathol. 2008;9:661-73.

54. Hoagland DR, Arnon DI. Growing Plants without Soil by The Water-Culture Method. 1938

55. Zhang C, Mapes BE, Soden BJ. Bimodality in tropical water vapour. Q J Roy Meteorol Soc. 2003:129:2847-66.

56. MacQueen J. Some methods for classification and analysis of multivariate observations. In: Proceedings of the Fifth Berkeley Symposium on Mathematical Statistics and Probability, Volume 1: Statistics; 1967. Berkeley, Calif: University of California Press; 1967. p. 281-97.

57. Lichtenthaler HK. Chlorophylls and carotenoids: Pigments of photosynthetic biomembranes. Meth Enzymol. 1987;148:350-82.

\section{Submit your next manuscript to BioMed Central and take full advantage of:}

- Convenient online submission

- Thorough peer review

- No space constraints or color figure charges

- Immediate publication on acceptance

- Inclusion in PubMed, CAS, Scopus and Google Scholar

- Research which is freely available for redistribution

Submit your manuscript at www.biomedcentral.com/submit 